\title{
The Function of Telomere Clustering in Yeast: The Circe Effect
}

\author{
S.M. Gasser, ${ }^{* \dagger}$ F. Hediger, ${ }^{*}$ A. TAddei, ${ }^{*}$ F.R. Neumann, \\ AND M.R. GARTENBERG \\ ${ }^{*}$ Department of Molecular Biology and Frontiers in Genetics NCCR Program, University of Geneva, CH-1211 \\ Geneva, Switzerland; "Department of Pharmacology University of Medicine and Dentistry of New Jersey-Robert \\ Wood Johnson Medical School, Piscataway, New Jersey 08854
}

Recent work has shown convincingly that telomeres of unicellular organisms, such as budding and fission yeast, Trypanosoma and Plasmodia, are spatially clustered in the nucleus. Live imaging (Heun et al. 2001b; Tham et al. 2001; Hediger et al. 2002b) and controlled in situ hybridization experiments (Gotta et al. 1996; Hediger et al. 2002a) in budding yeast indicate that telomere clusters are often adjacent to the nuclear envelope (NE), although they are distributed independently of nuclear pores (Fig. 1A). Rather than through pores, telomeres are bound through two redundant mechanisms: one that requires the end-binding complex yKu, and a second that makes use of the "partitioning and anchoring domain" (PAD; Ansari and Gartenberg 1997) of the silent information regulator Sir4 (Hediger et al. 2002b; Taddei et al. 2004). This domain, which is adjacent to the lamin-like carboxyl terminus of Sir4, binds specifically to the protein Esc1 (Establishes Silent Chromatin; Andrulis et al. 2002). Esc1 is large acidic protein localized along the inner nuclear membrane at interpore spaces (Fig. 1B) (Taddei et al. 2004). The anchorage mediated by the yKu heterodimer is not fully characterized, yet, when targeted to DNA, the $\mathrm{yKu} 80$ subunit is able to relocalize DNA to the nuclear periphery in a manner independent of Sir4 or Esc1 (Taddei et al. 2004). The efficiency of yKu80-mediated anchorage varies between $\mathrm{G} 1$ and $\mathrm{S}$ phases of the cell cycle, being stronger in G1. This mechanism may reflect a link between telomere anchoring and telomere end protection. In contrast to earlier reports (Galy et al. 2000; Feuerbach et al. 2002), we find that yKu does not anchor telomeres through interaction with the two Myosin-like proteins Mlp1 or Mlp2. Deletion of both $M L P$ genes has no effect on telomere position, telomere silencing, or the localization of silent information regulatory proteins in budding yeast (Fig. 2) (Hediger et al. 2002a,b). Nonetheless, Mlp proteins do have a minor effect on telomere length (Hediger et al. 2002a).

\section{BIOLOGICAL FUNCTIONS OF TELOMERE CLUSTERING}

In wild-type cells, the clustering of telomeres creates zones in which silent information regulatory proteins, Sir2,

${ }^{\dagger}$ Corresponding author. Current address: Friedrich Miescher Institute, Maulbeerstrasse 66, CH-4058 Basel, Switzerland. E-mail: susan.gasser@fmi.ch.
Sir3, and Sir4, accumulate (Gotta et al. 1996). These proteins form a complex that is involved in the repression of transcription in a domain-specific, rather than promoterspecific, manner. The complex binds chromatin through interaction with histone amino-terminal tails. A point mutation in histone H4 K16 can displace the Sir complex and compromise telomeric repression (Hecht et al., 1995). Whereas Sir2 is a universally conserved NAD (nicotinamide adenine)-dependent histone deacetylase, Sir3 and Sir4 are neither conserved nor display any recognizable enzymatic activity (for review, see Gasser and Cockell 2001). The complex is thought to bind cooperatively along the nucleosomal fiber, a process that can be counteracted by barrier proteins (for review, see $\mathrm{Bi}$ and Broach 2001) or by Sas2, a histone acetylase that targets histone H4 K16 (Kimura et al. 2002; Suka et al. 2002). It is assumed, but has not yet been shown, that Sir complex binding folds the nucleosomal fiber into a higher-order structure.

Colocalization of the Sir protein pools with subtelomeric sequences and silent-mating-type loci was demonstrated by Chromatin IP and by double FISH-IF (fluorescence in situ hybridization immunofluorescence) localization (Gotta et al. 1996; Strahl-Bolsinger et al. 1997). The binding of Sir proteins to nucleosomes in these telomeric foci correlates strongly with subtelomeric transcriptional repression, called TPE (Hecht et al. 1995, 1996). Recent studies presented below have shown that if one disperses Sir pools throughout the nucleoplasm by eliminating the telomere anchors yKu and Esc1, silencerflanked reporter genes can be silenced without being proximal to telomeres (Gartenberg et al. 2004; see below). Similar effects can be achieved by overexpressing Sir proteins, although this can also have toxic effects (Holmes et al. 1997).

\section{GENETIC DIVERSITY THROUGH TELOMERE- ASSOCIATED VARIEGATION}

Insight into the relationships between telomere clustering, anchoring, and silencing have emerged from studies of pathogens such as Plasmodium falciparum, Trypanosoma brucei, or Candida glabrata. In all three species, telomeres are grouped at the NE as they are in budding yeast (Chung et al. 1990; Freitas-Junior et al. 2000). Furthermore, orthologs of yeast Sir proteins have been identified in Plasmodia and Candida (Scherf et al. 
A
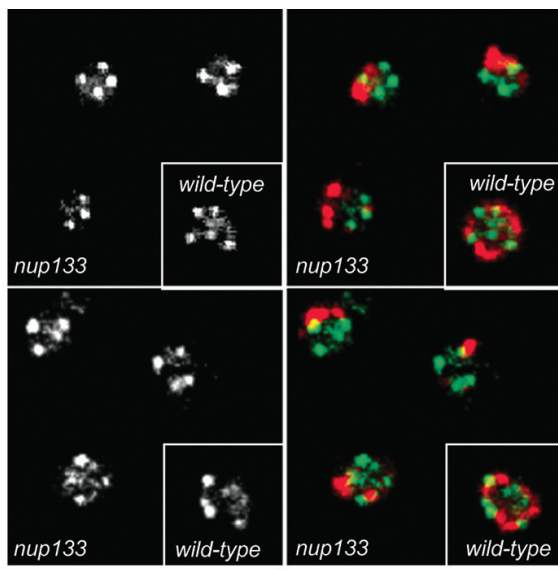

Rap1p Nup49
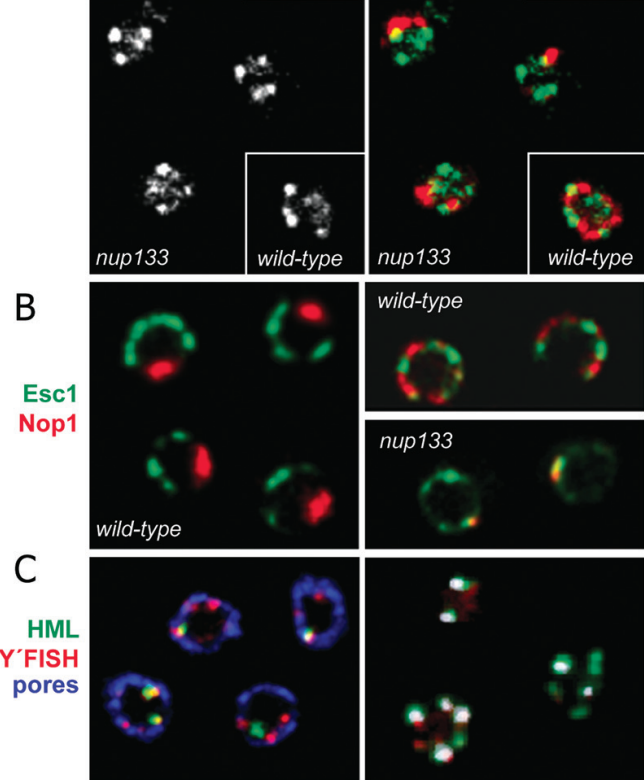

Figure 1. Subnuclear localization of telomeres, silencing factors, Esc1, and the silent $H M L$ locus. (A) Mutation of nup 133 does not affect telomere position, as detected by immunofluorescence (IF) for telomere-bound Rap1 and Sir4. IF was performed on fixed cells using affinity-purified polyclonal antibodies specific for Rap1 or Sir4, along with the monoclonal Mab414 (anti-pore). Cells were grown at $24^{\circ} \mathrm{C}$ and were prepared for IF as described in Heun et al. (2001a). Strains used were either wild type (shown in insets) or an isogenic strain deleted for nup 133. In the merged image, Rap1 or Sir4 signals are in green, while nuclear pore is in red. Overlap is in yellow. Bar, $2 \mu \mathrm{m}$. (B) Esc1 is localized at the nuclear periphery independently of pores (Taddei et al. 2004). Live visualization of endogenous Esc1GFP tagged and the nucleolar protein Nop1 (left panel), or the nuclear pore protein Nup49 (right panels) fused to CFP (shown in $r e d$ ) in a wild-type or nup 133 strain, as indicated. Shown are equatorial sections of nuclei after deconvolution of wide-field Zstacks of GFP and CFP signals. (C) Left: Two-color FISH with probes specific for the telomeric $\mathrm{Y}^{\prime}$ repeat $(\mathrm{red})$ and the silent $H M L$ locus in a diploid cell, which was arrested in G1 by alpha factor and counterstained with antinuclear pore Mab414 (blue). Shown are equatorial confocal sections of four cells. Labeling and microscopy as described in Heun et al. (2001a). Right: Colocalization of FISH for the telomeric Y' probe (red) and Sir3, detected with an affinity purified polyclonal antibody (green). Only $\sim 70 \%$ of foci colocalize (overlap in white; Gotta et al. 1996). (A, Reprinted, with permission, from Hediger et al. 2002a [CElsevier]; $B$, reprinted from Taddei et al. 2004 [CNature Publishing Group; http://www.nature.com/].)

2001; De Las Penas et al. 2003), increasing the likelihood for conserved anchoring and silencing pathways. In these pathogens, telomere clustering is linked to the organism's virulence (De Las Penas et al. 2003). High virulence in Plasmodia or Trypanosoma requires a sequential activation of variant-specific surface glycoprotein genes (vsg or var) at subtelomeric expression sites, allowing the para- site to escape the host immune system. The clustering of different var genes in groups, which correspond to telomeric foci, is thought to enhance recombination efficiency and thereby increases the parasites' virulence by increasing antigenic variability. Although telomere-anchoring proteins are not yet characterized, Figueiredo and colleagues showed that the clustering of Plasmodia telomeres depends on subtelomeric elements (Figueiredo et al. 2002). It is not known as yet whether virulence can be compromised by interfering with telomere clustering.

In the fungal pathogen, Candida glabrata, virulence depends on the adherence of the parasite to the intestine epithelia through the lectin EPA-1 (Cormack et al. 1999). EPA-1 is member of a family of related Candida genes found in subtelomeric clusters, most of which are not expressed at any given time. Their repression resembles the reversible position effect found at budding yeast telomeres and is mediated by homologs of the $\mathrm{ScSir} 3$ and ScRap1 proteins (De Las Penas et al. 2003). Highly analogous is a budding yeast gene family of FLO genes, which encode cell-wall glycoproteins involved in regulating cell adherence and a developmental transition. One family member, the FLO11 gene, is nonsubtelomeric, yet shows a variegated pattern of repression that requires a promoter-bound factor and the histone deacetylase Hda1 (Halme et al. 2004). This silencing does not require the SIR gene products. In contrast, FLO10, like most other $F L O$ genes, is located in a subtelomeric zone of reduced gene expression, called the HAST domain (ㅂda1-affected subtelomeric regions; Robyr et al. 2002). These genes show variegated, position-dependent repression that does require telomere-binding factors $\mathrm{yKu}$ and Sir3. Surprisingly, neither Sir4 nor Sir2 is implicated in this regulation, although the Sir2 homolog Hst1 is. The variegated expression of the yeast $F L O$ genes is highly reminiscent of mechanisms used to ensure phenotypic variation of cell surface markers in pathogenic microorganisms, like Plasmodia, Trypanosoma, and Candida. Thus we propose that unicellular organisms may use the perinuclear clustering of telomeres and its associated recombination and variegation phenotypes to expand their repertoire of gene expression patterns improving survival in adverse situations.

\section{SILENT CHROMATIN DIRECTS ITS OWN ANCHORAGE INDEPENDENTLY OF A CHROMOSOMAL CONTEXT}

Our studies of yeast telomere anchoring raised the following question: Does silent chromatin that has no chromosomal end nearby also associate with the nuclear periphery? Does repressed chromatin per se have a subnuclear "address"? Because the targeting of a Sir 4 PAD fusion relocalizes an internal chromosomal domain to the NE (Taddei et al. 2004), we hypothesized that silent chromatin may be anchored directly. We therefore tested whether full-length Sir4, as an integral component of silent chromatin, could also mediate anchorage of a chromosomal domain.

In situ hybridization studies showed that the two silent yeast mating-type loci, $H M L$ and $H M R$, which are posi- 
A

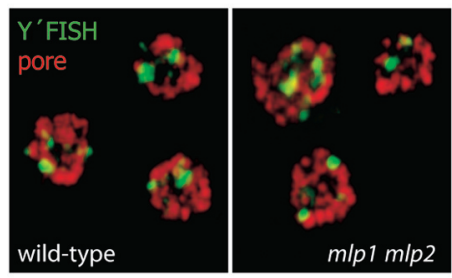

B

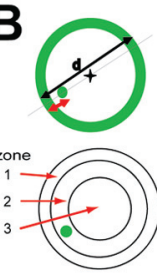

C

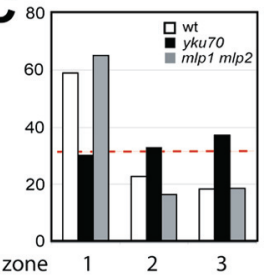

D
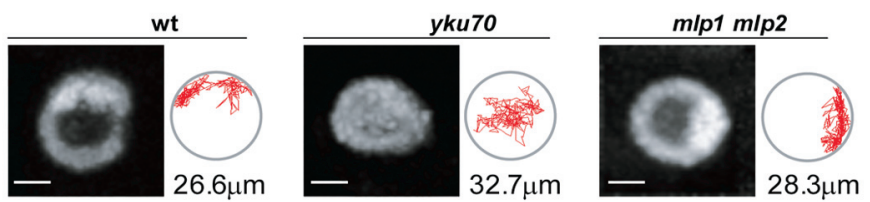

E
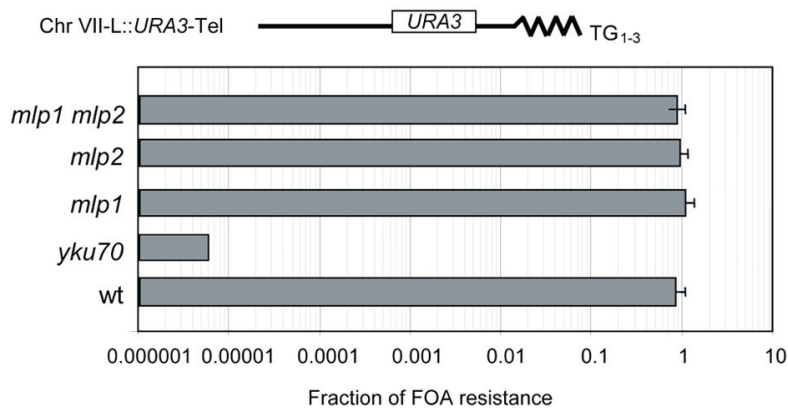

Figure 2. MLP1 and MLP2 deletion does not alter telomere distribution nor TPE. (A) In situ hybridization with a probe recognizing $\mathrm{TG}_{(1-3)}$ and $\mathrm{Y}^{\prime}$ sequences and immunofluorescence with affinity-purified polyclonal antibodies specific for Sir4 were performed on wild-type LM11 (wt; Maillet et al. 2001) and an isogenic strain deleted for $m l p 1$ and $m l p 2$ (GA1471, $\mathrm{mlp} 1 \mathrm{mlp2}$ ). Telomeres are in green, while nuclear pore staining (Mab414) is in red. Shown are confocal images as published in Hediger et al. (2002a) showing equatorial sections of yeast nuclei that are intact after the staining procedure. Spread or flattened nuclei were not analyzed. Bar, $2 \mathrm{~mm}$. $(B)$ A scheme showing an equatorial section of fluorescence for cells bearing Nup49-GFP and a tagged genomic locus. Distances between the lacI-GFP-tagged locus and Nup49-GFP at the nuclear envelope (x) are normalized to the nuclear diameter (y) and binned according to three zones of equal surface area. In a $2-\mu \mathrm{m}$-diameter nucleus, zone 1 corresponds to the outermost ring of $\sim 190 \mathrm{~nm}$ width. Random distribution implies $33 \%$ in each zone. (C) Telomere $6 \mathrm{R}$ is not delocalized in the mlp $1 m l p 2$ mutant. Position of telomere 6R in G1 cells is represented in bar graphs as percentage of spot ( $y$ axis) per zone ( $x$ axis) for wild type, $y k u 70$, or $\mathrm{mlp} 1 \mathrm{mlp} 2$ strains (from Hediger et al. 2002b). Values $>33 \%$ show an enrichment of Tel6R at the nuclear periphery. Cells analyzed are wt, $n=110$; $y k u 70, n=168$; for $m l p 1 m l p 2, n=124$. (D) 250 sequential confocal images (at 1.5-sec intervals) from a typical time-lapse series of G1 phase nuclei bearing the Tel 6R tag were first aligned based on their nuclear pore signals, and then the absolute position of focus was marked using the AIM tool of the Zeiss LSM 510 software (rel. 2.8). The locus' trajectory is projected in red on a single focal section of the nucleus. Strains used are wild-type (GA-1459), a yku70 deletion (GA-1489), and the mlp1 mlp2 double deletion (GA1731). The mean length of the path in millimeters for a 5-min movie (200 frames) averaged over eight movies is indicated at the lower right of each image (for details, see Hediger et al. 2002b). (E) $\mathrm{mlp} 1 \mathrm{mlp} 2$ mutants show normal levels of repression at telomeres. The wild-type LM11 background containing the URA3 reporter at Tel VII-L and the same strain with either $\mathrm{mlp} 1, \mathrm{mlp} 2$, or $\mathrm{mlp} 1 \mathrm{mlp} 2$ $(\mathrm{mlp} / 1 / 2)$ deletions were grown overnight in YPAD, and tenfold serial dilutions were plated onto synthetic complete medium (SC) and SC containing $0.1 \%$ 5-FOA. Similar results were obtained for the ADE2 gene on TelV-R and for URA3 in another strain background (Hediger et al. 2002a). (A,E, Reprinted from Hediger et al. 2002a [C Elsevier]; D, reprinted from Hediger et al. 2002b [CElsevier].)

tioned within $25 \mathrm{~kb}$ of telomeres, are indeed found at or near the NE, and $H M L$ was frequently found clustered with other telomeres (Fig. 1C) (Laroche et al. 2000). The contour length of DNA sequence (in base pairs [bp]) separating the telomeres and silent loci is below the resolution of the light microscope. Thus, it is impossible to determine whether or not $H M$ loci are simply held at the nuclear periphery because of anchorage of the adjacent telomere. To eliminate the influence of cis-linked telomeres on silent chromatin localization, we used inducible site-specific recombination to uncouple the HMR locus from its normal chromosomal context (Fig. 3A) (Cheng et al. 1998). A genomic region of $17 \mathrm{~kb}$, containing $H M R$ with its silencers and an integrated lac operator array $\left(l a c^{o p}\right)$, was flanked by a pair of target sites for the $\mathrm{R}$ site-specific recombinase. Galactose-induced expression of the recombinase yielded $95 \%$ excision within 2 hours in a silencing-competent strain. The locus could be monitored relative to GFP-tagged nuclear pores and, by comparing strains that had or had not undergone excision, the impact of chromosomal context on location and dynamics of $H M R$ could be evaluated (Fig. 3).

In a strain that lacks recombinase (SIR+/Rec-), the unexcised $H M R$ remains closely linked to the nuclear envelope (Fig. 3B). In a silencing-defective strain (sir3 deletion; sir $3 / \mathrm{Rec}-$ ), the perinuclear fraction of HMR dropped only slightly ( $91 \%$ to $81 \%$; Fig. 3B). This was expected, since a yKu-dependent mechanism is known to anchor 
telomeres in the absence of silencing (Hediger et al. 2002b). Intriguingly, when HMR was uncoupled from the chromosome as an excised ring, it remained at the nuclear envelope in $86 \%$ of silencing-competent cells (SIR+/Rec+; Fig. 3B). This shows that HMR association with the nuclear periphery is an intrinsic property of the locus and does not depend on linkage to a telomere. Furthermore, when the ring was excised in a sir3 deletion strain, it lost anchorage (sir3/Rec+; Fig. 3B). The unconstrained mobility of a nonsilent ring can be demonstrated by time-lapse tracking (Fig. 3C; see 5-min trajectories of excised rings). This allows us to conclude that immobilization of the excised HMR locus at the NE depends on its silent state, unlike the situation at telomeres.

\section{Two Types of Perinuclear Anchorage: Independent of and Dependent on Silencing}

Because yeast has no intermediate filament proteins like the nuclear lamina, and because neither yKu nor Sir proteins have membrane-spanning domains, we screened for $\mathrm{NE}$ components that tether $\mathrm{yKu}$ and silent chromatin. By exploiting an in vivo chromatin relocalization assay (Fig. 4A) (Taddei et al. 2004), we have been able to assay protein domains for their ability to direct chromatin to the nuclear periphery independently of their interaction with silencing factors. As shown here (Fig. 4B), minimal protein domains that are sufficient to relocate a tagged locus to the NE include $\mathrm{yKu} 80, \mathrm{Sir} 4^{\mathrm{PAD}}$, the carboxy-terminal domain of Esc1, and a transmembrane protein like Yif1 (Taddei et al. 2004). Esc1 interacts with Sir4 and is required for the stable mitotic partitioning of a Sir4-bound plasmid (Andrulis et al. 2002). Importantly, as Esc1 is positioned at the nuclear periphery in strains lacking $Y K U$ and SIR genes, it appears to be a structural element that does not require chromatin for its localization. Nonetheless, Esc1 contributes to efficient telomeric silencing (Taddei et al. 2004). Most importantly, the relocalization of a chromatin domain through the targeting of the Sir4 ${ }^{\mathrm{PAD}}$ domain requires either Esc1 or $\mathrm{Ku}$ (Fig. 4C,D), arguing that these ligands constitute necessary but redundant pathways for the anchoring of both telomeres and silent chromatin by Sir4.

Mlp1 and Mlp2 were previously proposed to form a bridge between yKu and nuclear pores (Galy et al. 2000). If this were true, telomeres would be released from their perinuclear anchorage in the double $\mathrm{mlp} 1 \mathrm{mlp} 2$ deletion strain, similar to their delocalization in $y k u$-deficient strains. Based both on an analysis of live cells and on FISH studies, this is not the case: As shown above, telo-

A

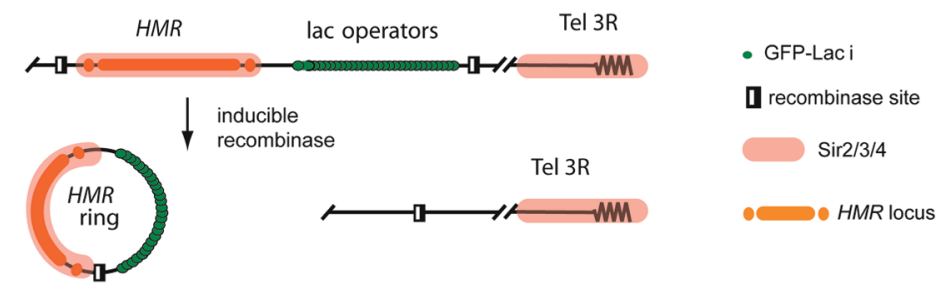

B

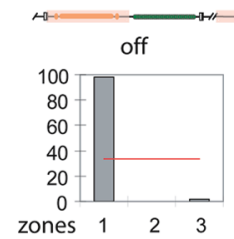

HMR chromosomal

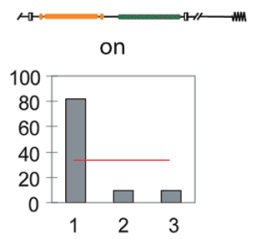

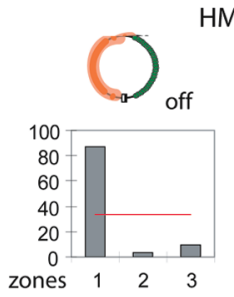

HMR ring

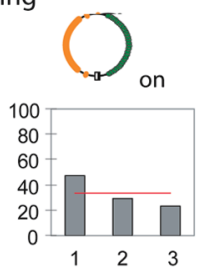

C

HMR chromosomal

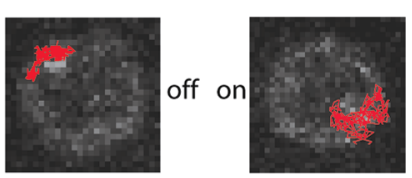

HMR ring

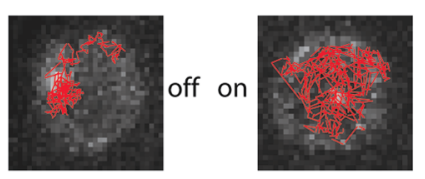

5-min trajectories in real time

Figure 3. Telomere independent anchoring of silent chromatin. (A) Site-specific recombination at engineered target sites (RS sites) uncouples the lac ${ }^{\mathrm{op}}$-tagged $H M R$ locus from the chromosome. The strains carry Lac-GFP, Nup49-GFP, and a galactose-inducible R recombinase, and are either competent for repression ( $\mathrm{Sir}+$ ) or deficient (sir3 deletion). (B) Distribution of HMR in the three zones is plotted as a percentage of the total number of cells counted for each strain, as described in Fig. $2 \mathrm{~B}$. Zone 1 is the outermost zone and $33 \%$ would be a random distribution. Strains used and cells counted are MRG2253 SIR+/chromo, $n=140$; MRG2251 sir3/chromo, $n=232$; MRG2249 SIR+/ring, $n=173$; MRG2250 sir3/ring, $n=192$. (C) The trajectory of the tagged HMR locus is traced in a representative cell from each relevant strain. A 3D stack of images was taken at 1.5 -sec intervals for 5 min and projected onto one plane for tracking and quantitation (see Gartenberg et al. 2004). 

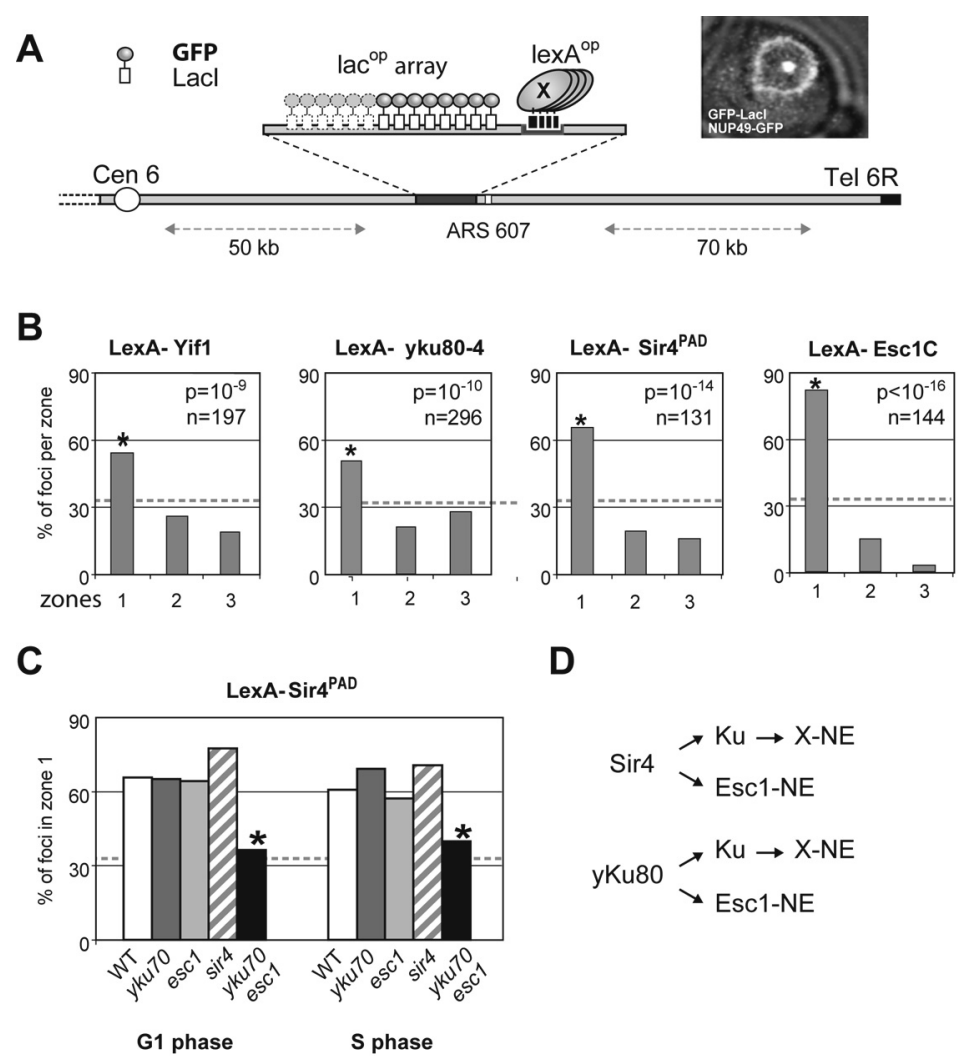

D

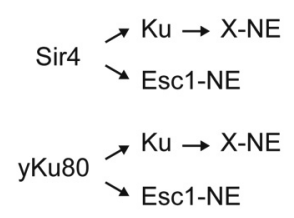

Figure 4. A chromatin relocalization assay identifies yKu- and Sir4-dependent anchoring pathways. $(A)$ A yeast strain bearing lac ${ }^{o p}$ repeats and LexA binding sites at an internal locus on the right arm of Chr 6 (Chr6 $\left.{ }^{\text {int }}\right)$ and a GFP-Nup49 fusion is transformed with plasmids expressing different LexA-protein fusions (Taddei et al. 2004). The position of the locus is scored as in Fig. 2B. (B) LexA.Yif1, LexA-Sir4 ${ }^{\mathrm{PAD}}$ LexA-yku80, and LexA-Esc $1^{\mathrm{C}}$ are able to relocate the locus to the NE. Bar graphs present the percentage of spots ( $y$ axis; $n=$ number of cells analyzed) per zone ( $x$ axis). $P$-values are calculated by comparing actual proportion of cells bearing a spot within zone 1 to a hypothetical random distribution. $(C)$ LexA-Sir4 ${ }^{\mathrm{PAD}}$ was expressed in GA-1461 bearing complete disruptions of $y k u 70, e s c 1, \operatorname{sir} 4$, and $y k u 70$ escl, as indicated. Chr $6^{\text {int }}$ position was scored as in Fig. 2B, and zone 1 values are compared among strains for G1 and S phase (for statistics, see Taddei et al. 2004). The black* indicates that values are indistinguishable from a random distribution $(p>0.05)$. LexA-Sir $4^{\mathrm{PAD}}$ chromatin relocation activity was unaffected by loss of either Esc1 or yKu70 individually, yet we see a complete loss of the Sir ${ }^{\mathrm{PAD}}$ tethering activity in the absence of both Esc1 and $\mathrm{Ku}$, throughout the cell cycle. This result strongly suggests that Esc1 and Ku provide two parallel anchorage pathways for Sir4 ${ }^{\mathrm{PAD}}$. $(D)$ We show the dual pathways of anchoring mediated by Sir4-Esc 1 and by Ku through an unknown protein (x) at the nuclear envelope, which allows relocalization of internal sequences in the absence of silencing. During G1 phase in wild-type yeast cells the yKu-mediated pathway appears to be favored while the Sir-Esc1 anchoring pathway functions efficiently in S phase (for details, see Taddei et al. 2004). (Reprinted, with permission, from Taddei et al. 2004 [@Nature Publishing Group; http://www.nature.com/].)

meres remain completely anchored in the double $m l p l$ mlp2 mutant based on both live imaging and $\mathrm{Y}^{\prime}$ FISH (Fig. 2A,C). Moreover, while some but not all telomeres are released from the periphery in the $y k u 70$ single mutant, we could show that all lose anchorage in $y \mathrm{ku} 70 \mathrm{escl}$ double mutants (Fig. 5A,B). Thus, the anchoring from $\mathrm{yKu}$ and from Sir4PAD-Esc1 provides parallel mechanisms that tether telomeres to the NE. Intriguingly, different telomeres show a differential dependence on the two pathways, possibly reflecting the efficiency of subtelomeric heterochromatin formation (Fig. 5A,B) (Tham et al. 2001; Hediger et al. 2002b; Taddei et al. 2004).

\section{TESTING THE ROLE OF PERINUCLEAR ANCHORING IN REPRESSION}

It has been proposed that the anchorage of telomeres at the nuclear periphery sequesters silencing factors and thereby promotes repression of adjacent genes (Maillet et al. 1996). This model is based on several observations: The first is that normal cellular Sir protein concentrations are limiting for repression. This notion was supported by the observation that balanced overexpression of Sir2, Sir3, and Sir4 (Maillet et al. 1996) or of Sir3 alone (Renauld et al. 1993) improves TPE, although $H M$ repression appears to be resistant to this variability. Moreover, mutations were also discovered that enhance the ability of the $H M$ loci and telomeres to compete with one another for Sir factors (Buck and Shore 1995). Second, we know from the work of several laboratories that silencerflanked reporter genes are more efficiently repressed when inserted near the telomere. Although this shows that linear distance from a telomere can influence repression, it was unclear whether this reflected a positive effect of the nuclear envelope on repression or an effect of Sir factor concentration in telomeric pools. Indeed, the spatial or subnuclear position of these translocated silencing cassettes was never determined. Third, it was shown that 
A
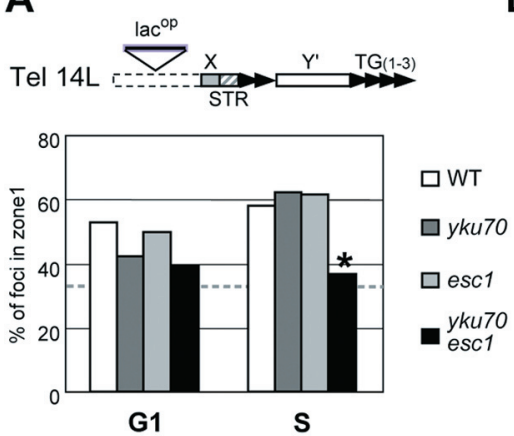

C

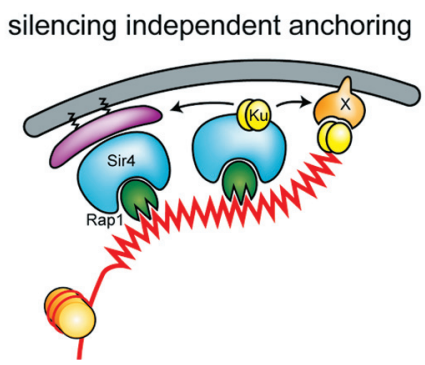

B
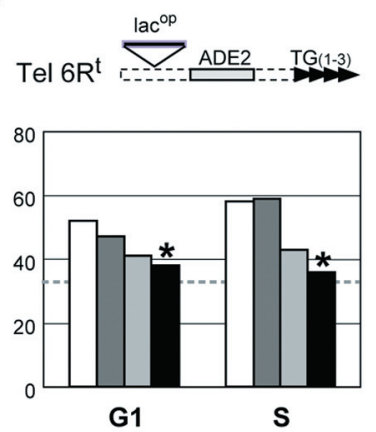

silencing dependent anchoring

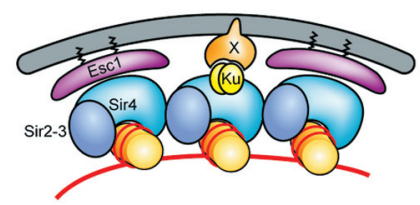

Figure 5. YKu and Esc1 anchor yeast telomeres in vivo. (A) Tel 14L position was determined in G1 and S phase cells of GA-1985, yku70 (GA-1983), escl (GA-2074), and yku70 escl (GA-2082) strains, as in Fig. 4. For statistics see Taddei et al. (2004). (B) Truncated Tel 6R ${ }^{t}$ localization was determined in WT (GA-1917), yku70 (GA-1918), escl (GA-2229), and yku70 escl (GA-2230) strains as in A. (C) Scheme of dual telomere anchoring pathways: one through Escl and the other through yKu. This can occur for natural telomeres through yKu evening the absence of silencing (Hediger et al. 2002a; Taddei et al. 2004). In the presence of silent chromatin, Sir4 is available to bind $\mathrm{Esc} 1$ and $\mathrm{yKu}$, thereby anchoring repressed chromatin in the absence of a nearby telomere. (Reprinted, with permission, from Taddei et al. 2004 [CNature Publishing Group; http://www.nature.com/].)

the delocalization of Sir factors from telomeres, provoked either by mutation or overexpression, was able to restore repression at loci distant from telomeres (Lustig et al. 1996; Maillet et al. 1996, 2001; Marcand et al. 1996). This confirms that telomeric foci sequester repressors from other sites of action. Again, it was not shown whether the "internal" silenced loci relocate to the periphery once repressed. Such analysis is complicated by the fact that internal repression efficiency is often low.

In support of a functional role for telomere anchoring, the Sternglanz laboratory showed that the artificial "tethering" of a reporter gene to the yeast NE through a membrane-spanning protein or motif could favor transcriptional repression (Andrulis et al. 1998). This required the presence of at least one silencer element, a specific cisacting sequence that nucleates Sir-dependent repression. It was proposed that this NE "tether" would promote repression because it places the weak silencer near a zone of high Sir protein concentration. However, other interpretations were not only possible, but likely. Notably, three results suggested that the nuclear periphery contributes to repression through something other than an increase in proximity to Sir proteins. First, Andrulis et al. (1998) showed that overexpression of Sir proteins did not obviate the positive effect conferred by the transmembrane tethering of the reporter construct. Thus, it would seem that the NE provides something necessary for repression in addition to the high Sir concentrations. Con- sistently, Galy et al. (2000) argued that loss of the poreassociated proteins Mlp1 and Mlp2 disrupted telomeric silencing and telomere anchoring. Although Mlp proteins are not needed for telomeric repression (Fig. 2E) (Andrulis et al. 2002), the notion that perturbation of nuclear pore architecture influences repression is often repeated (Feuerbach et al. 2002). From these arguments arose the notion that the nuclear envelope/pore environment is needed to promote repression.

Making use of the excised $H M$ ring described in Figure 3 , we were able to test this hypothesis. Knowing that Sir4 ${ }^{\mathrm{PAD}}$ can anchor through both yKu and Esc1, we examined the locus dynamics and the repression status of the excised $H M R$ ring in a strain lacking the two anchorage pathways. We ask whether the double esc $1 \mathrm{ku} 70$ deletion would release the $H M R$ ring from its perinuclear anchorage and, if so, whether this would compromise silencing. Although telomeric repression is eliminated by the deletion of yKu subunits, $y k u 70$ deletion does not weaken mating repression at the endogenous $H M L$ (Laroche et al. 1998) or HMR loci (Boulton and Jackson 1998; Mishra and Shore 1999) to any significant degree. To monitor repression at the silent mating-type loci, the active MAT locus was deleted in these strains and the transcriptional state of the $a l$ gene at $H M R$, the sole remaining copy of this gene, was determined by northern blot analysis. We note that although al mRNA was repressed as expected in the single $k u 70$ and esc 1 mutants 
and derepressed in the sir3 strain, it was surprisingly completely silent in the esc 1 yku70 double mutant, irrespective of whether HMR was excised or not (Fig. 6A) (see also Gartenberg et al. 2004).

We then monitored the dynamics of the silent $H M R$ ring in the escl yku70 mutant. Rapid time-lapse imaging of the ring in intact cells reveals that the excised HMR locus is completely mobile, showing no preferential association with the nuclear envelope. Its dynamics and radii of constraint (for description of imaging and Mean Squared Displacement analysis, see Gartenberg et al. 2004) are indistinguishable from a nonsilent ring (Fig. 6B). This al- lows us to functionally separate silencing from perinuclear anchorage, and to conclude that the NE per se is not necessary for maintenance of the repressed state.

At the same time we have probed for Sir protein distribution by immunostaining. Sir3 and Sir4 are almost completely dispersed from subtelomeric foci in the escl $y k u 70$ double mutant, while they remain largely perinuclear when only escl or yku70 is deleted (Fig. 6C for Sir4) (Gartenberg et al. 2004). Thus, Sir-mediated repression does not have to occur at the nuclear envelope, at least when Sir proteins are no longer sequestered by telomeres. This does not argue, however, that Sir pools

A

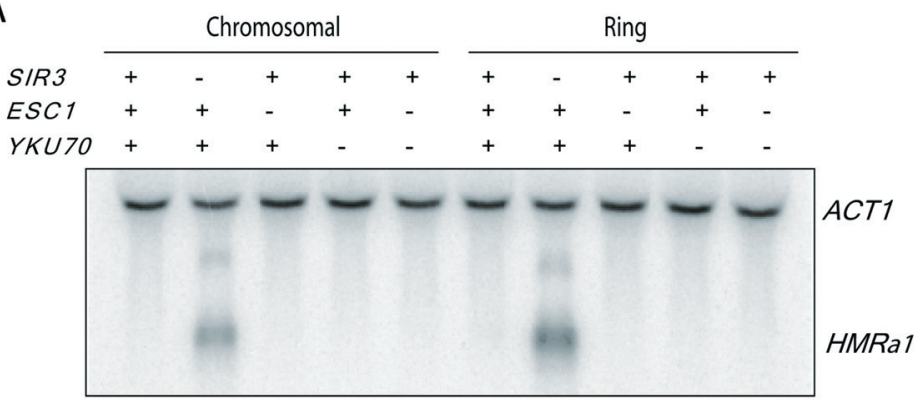

B

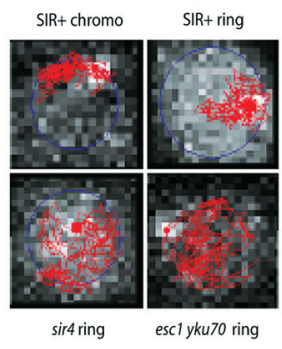

Mean Square Displacement Analysis of HMR rings

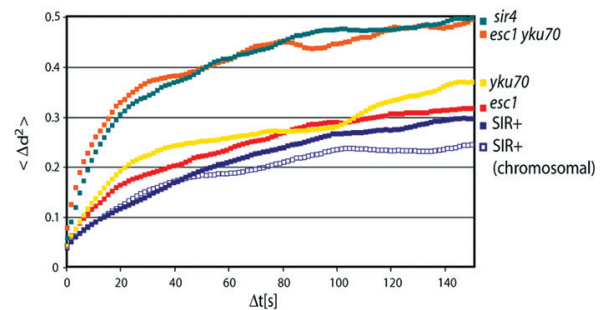

C
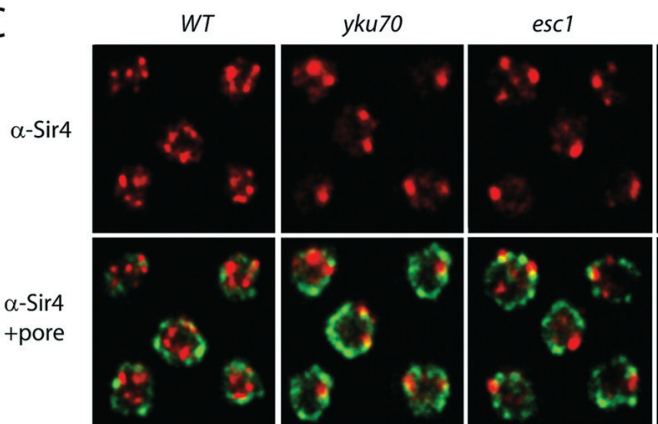

esc1 yku70

Figure 6. (A) The excised $H M R$ ring remains transcriptionally silent. Cultures were grown to induce excision of the $H M R$ locus as shown in Fig. 3. Samples were harvested immediately before galactose addition (labeled chromosomal) or $4 \mathrm{hr}$ thereafter (labeled ring). Lanes 1 and 6, MRG2262 SIR+; lanes 2 and 7, MRG2263 sir3; lanes 3 and 8, MRG2264 esc1; lanes 4 and 9, MRG2265 yku70; lanes 5 and 10, MRG2266 esc1 yku70. Blot was hybridized simultaneously with probes to the $\boldsymbol{a} 1$ gene at $H M R$ and $A C T 1$ as an internal control (data from Gartenberg et al. 2004). (B) The 5-min trajectories of the movement of the excised HMR ring in MRG2197 SIR+/chromo, MRG2201 SIR+/ring, MRG2267 sir4/ring, and MRG2255 esc1 yku70/ring. Images were taken as 3D stacks every 1.5sec over a 5-min interval, and are projected onto one plane for tracking and quantitation. Mean Squared Displacement analysis of the excised $H M R$ ring was performed by computing the square of the displacement, $\Delta d^{2}=[d(t)-d(t+\Delta t)]^{2}$, over increasing time intervals 1.5-150-sec; see Gartenberg et al. 2004). The average of all $\Delta d^{2}$ values for each $\Delta t$ value was plotted against $\Delta t$. Over 65 min of projected 3D stacks taken at 1.5 -sec intervals are analyzed per strain. All data are after induced excision except SIR + chromosomal, which shows the constraint of mobility imposed by the chromosomal fiber and the repressed state. (C) Immunolocalization of Sir4 (red) and nuclear pore (green) on strains used in $A$ and $B$. Affinity-purified anti-Sir4 rabbit serum and Mab414 (mouse anti-pore) were applied after growth and fixation with formaldehyde in YPD (Gartenberg et al. 2004). 
have no role in repression in a wild-type situation. Rather, one could argue that a local increase in Sir protein concentration may be the only essential contribution of the nuclear envelope to repression, since silent chromatin is maintained internally after ablation of the two perinuclear anchors for Sir4. We propose that if critical Sir concentrations are achieved by other means, the NE will have no further role in repression. This does not, however, rule out a possible role for the NE in nucleation events. Indeed, the weaker the silencer, the more important positioning at the NE and proximity to telomere clusters will be.

These results also provide a plausible explanation for why improved silencing in wild-type cells correlates with improved anchoring (Tham et al. 2001; Hediger et al. $2002 b$ ). Because silent chromatin can itself target the repressed locus to the periphery (as long as either $\mathrm{yKu} 80$ or Esc1 is present), then silent domains will accumulate at the periphery as a result of repression. Ironically, the general limitation on Sir protein availability, which is the reason one cannot repress internally, stems in part from the sequestering of Sir proteins by telomeres. This chickenand-egg situation is resolved by noting that telomere anchoring at the NE can occur in the absence of silencing, providing a means to initiate the formation of these repressive subcompartments. Thus telomere anchoring probably precedes repression.

A corollary to these findings is the expectation that relocalization to the NE alone will not be sufficient to silence a locus. Support for this, data showing that perinuclear anchoring can occur without silencing, comes from many sources. First, Andrulis et al. (1998) showed that reporter gene anchoring through a membrane protein did not repress the reporter if no silencer was present. Second, truncated telomeres remain fully anchored in $y k u$ mutants, although TPE is compromised by $10^{4}$-fold (Tham et al. 2001) and internal sequences can be dragged to the nuclear envelope by targeted protein domains, in the absence of silencing (Taddei et al. 2004). This conclusion was actually evident from the very first descriptions of TPE and telomere anchoring. Gottschling et al.
(1990) showed that subtelomeric silencing is stochastic and occurs at a frequency ranging from $0.5 \%$ to maximally $30 \%$ of cells (depending on the reporter and the telomere), while telomeres are on average about $70 \%$ tightly anchored (Gotta et al. 1996). Further, in a sir-deficient strain, most telomeres remain at the periphery despite the fact that there is no silencing. It follows that transcribed telomeres are able to remain anchored, a phenomenon we now know to be mediated by yKu (Hediger et al. 2002b; Taddei et al. 2004).

\section{SPONTANEOUS AND SELF-PROPAGATING Sir POOLS}

These results lead to a model that explains how a Sirrich compartment can form spontaneously in the nucleus (Fig. 7). Central to this model are two facts: Silencing-independent anchorage can be achieved through yKu (and possibly also Sir4; see Fig. 5C), and silencing-dependent anchorage occurs through Sir4. The yKu-mediated anchoring provides a means for telomeres to accumulate at the NE prior to the assembly of repressed chromatin. The anchoring of telomeric repeats creates a large number of potential Sir4 protein binding sites within a restricted volume, because of the presence of 20-25 Rap 1 consensuses on each telomere end (Gilson et al. 1993), and the ability of Rap1 to bind both Sir3 and Sir4 (Moretti et al. 1994). The telomere-associated yKu dimer also binds Sir4 tightly (Roy et al. 2004). Subtelomeric repeats could further contribute to the efficiency of Sir4 accumulation through ORC- and Abf1-binding sites. The resulting high density of Sir4-binding chromatin would in turn recruit Sir2 and Sir3, again through protein-protein interactions (Moretti et al. 1994; for review, see Gasser and Cockell 2001). Once repressed chromatin is nucleated, spreading would be favored by the high local concentration of silencing factors. As silent chromatin spreads, it augments the amount of stably bound Sir complex and in turn reinforces the interaction of the telomere (or of another locus) with the NE. This creates a feedback loop, which can be initiated by binding a very limited number of yKu or Sir4

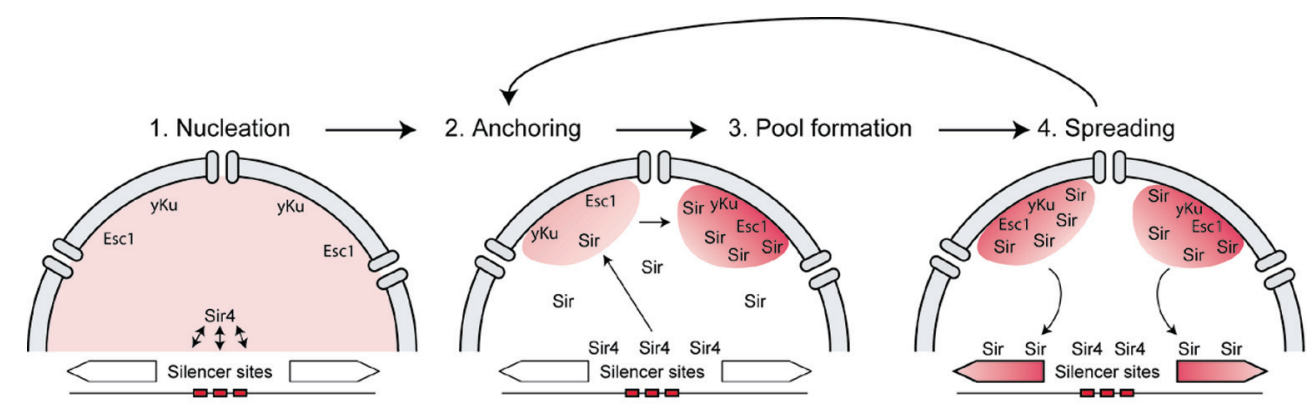

Figure 7. Model for the role of chromatin anchoring in the promotion of silencing. We propose that Sir4 is first recruited at the nucleation center by DNA binding proteins that can also bind Sir proteins (step 1; Nucleation). These include Rap1, ORC, Abf1, and $\mathrm{yKu}$. The presence of Sir4 at the locus will then bring it to the nuclear periphery through one of the two Sir4 anchoring pathways (step 2; yKu or Esc1) where the high local concentrations of Sir proteins will be created, in part by the clustering of telomeres (step 3). This, in turn, helps silencing complexes assemble and spread (step 4). The anchoring of silent loci at the periphery will increase the concentration of Sir proteins and reinforce the silencing of other loci within this region. This can be self-propagating. In addition, the multiple telomere-associated Rap1 sites create a sink that maintains a pool of Sir factors for incorporation into repressed chromatin--the so-called "Circe effect." 
proteins at given site (summarized in Fig. 7). It is not clear what the minimal number of anchoring molecules may be, but in a targeting assay, four double lexA sites were sufficient (Taddei et al. 2004). We envision such mechanisms as also being relevant for the clustering of centromeric satellite and heterochromatin sequences in higher eukaryotes, which may similarly promote the sequestration of heterochromatin factors like HP1.

\section{THE CIRCE EFFECT: CREATING COMPARTMENTS FOR GENERAL REPRESSORS}

This apparent sequestration of Sir proteins in telomeric pools can be described by a term from enzymology - the "Circe effect." (In Greek mythology, Circe was an enchantress who detained Odysseus on her island and turned his men into swine. Jencks [1975], a brilliant enzymologist, defined the Circe effect as "the utilization of strong attractive force to lure a substrate into a site in which it undergoes an extraordinary transformation.") This term was initially used to describe the attractive effect that weak nonproductive binding sites would have on the association of a substrate with the active site of an enzyme (Jencks 1975). The presence of numerous "nonproductive" binding sites are described as "luring" the substrate into the active site of an enzyme, where it would undergo transformation "in form and structure." It could be argued that the substrate would not be in equilibrium with its concentration as determined for a large pool (the nucleus), but would be in equilibrium with a local volume that is enriched for the ligand, because of the spatial proximity of multiple weak interaction sites. Here we apply this notion to describe how the pools of "nonengaged" Sir proteins held by clustered yeast telomeres and their multiple Rap1 sites might favor the assembly of a repressed chromatin state. Once Sir proteins form a stable complex with chromatin, we argue they are "transformed in form and structure."

To apply this concept to general repressors such as Sir proteins we would have to postulate first that Sir proteins are limiting for assembly into silent chromatin. The welldocumented effects of Sir protein overexpression are consistent with this idea (Renauld et al. 1993; Maillet et al. 1996). Second, we need to postulate that there are two types of binding sites for Sir proteins. Again this seems to be the case. There are weak, unstable interactions of Sir3 and Sir4 with Rap1, which itself binds with high affinity to the TG repeats at telomeres. The instability of Rap1-Sir interactions is attested by the fact that these proteins cannot be immunoprecipitated without cross-linking reagents, whereas the SIR-nucleosome complexes can be. The average spacing of one Rap 1 site per $18 \mathrm{bp}$ within the TG repeat leads to 20-25 weak binding sites for Sir3 and Sir4 per chromosomal end. Assuming that telomeres cluster in groups of 6 , this results in 180 weak binding sites. Indeed, ChIP (chromatin immunoprecipitation) data indicate that Sir4 can be recovered with telomeres in the absence of silencing (Luo et al. 2002). We would argue that within a given focus of telomeres, the Sir proteins stay sequestered even when they are not incorpo- rated into repressive chromatin, thanks to their weak affinity for Rap1. This is further supported by the fact that Rif1 and Rif2 compete for Sir interaction with Rap1, and that the loss of Rif proteins improves silencing (Mishra and Shore 1999). The second type of "Sir interaction" that occurs in telomeric foci would be that resulting from the incorporation of the complex into a higher-order chromatin structure. FRAP (fluorescence recovery after photobleaching) experiments show this interaction to be much more stable than that of Rapl with DNA (T. Cheutin et al., unpubl.). We note further that the assembly of Sirs into silent chromatin may be cooperative. Although this remains to be proven, the linear spreading of silent chromatin would be consistent with cooperativity in the assembly of this higher-order structure (Renauld et al. 1993). For a cooperative event, local reserves of Sir proteins should indeed favor repression. The fact that unlinked or imbalanced Sir2 or Sir4 expression derepresses TPE suggests that factor dosage is also important within the complex or else that secondary, nonproductive complexes can be formed by two of the three Sir partners.

Given the different types of binding sites, one being more stable than the other, and the silencing-independent clustering of yeast telomeres, it would seem that the "Circe effect" may indeed accurately describe the sequestering of Sir proteins in perinuclear pools by telomeric repeats. These then serve to favor the formation of repressed chromatin, which is proposed to occur in at least two steps (Hoppe et al. 2002), the sum of which may well be considered equivalent to "transformation by an enzyme's active site."

Why should a cell bother to create repressive subcompartments, when the esc1 yku70 mutant shows that release of Sirs allows silencing without specific localization? We propose that telomere anchoring, and its encumbant sequestering of Sir factors, serves to prevent promiscuous repression throughout the rest of the active genome. The creation of subcompartments enriched for Sir proteins allows the cell to ensure that most other loci remain active, while nonetheless exploiting the power of a general, promoter-independent, transcriptional repression mechanism. This protective function has also been proposed for the methylation of lysine 79 in histone H3, a modification that is found throughout the active genome and that appears to be incompatible with silencing (van Leeuwen et al. 2002). Similarly the histone variant Htz1 is thought to help insulate the rest of the genome from inappropriate silencing events (Meneghini et al. 2003). By creating subcompartments that favor repression through the sequestering of Sir proteins, the cellular concentration of these general repressors can be maintained at relatively low levels to protect the genome from the deleterious action of runaway repression.

\section{EXPERIMENTAL PROCEDURES}

\section{Strain Construction and Yeast Techniques}

All strains are described elsewhere in the cited references, including Gartenberg et al. (2004), Hediger et al. (2002a,b), and Taddei et al. (2004). Standard techniques 
were used for immunofluorescence, FISH (Laroche et al. 2000; Hediger et al. 2004), Northern analysis, and silencing assays.

\section{Single Z-Stack Microscopy and Time-Lapse Analysis}

For the excision experiments, freshly streaked cells grown in SC-trp media containing 2\% dextrose were diluted into SC-his $+2 \%$ raffinose. When cultures reached $\sim 0.25 \mathrm{OD}_{600}$ after well-aerated overnight growth, galactose was added to $2 \%$. After 2 hours, $1 \mathrm{ml}$ of cell culture was harvested by centrifugation and either placed on microscope slides bearing $1.4 \%$ agarose plugs containing either $4 \%$ galactose $(\mathrm{pH} 5.8$ ) or $4 \%$ dextrose $(\mathrm{pH} \mathrm{7.0)}$ ), as needed, or were mounted in a Ludin chamber flushed with appropriate media (Hediger et al. 2004). Data collection was limited to 2 hours after mounting. For position data, Z-stacks were collected on an Olympus IX70 fluorescence microscope coupled to a TillVision imaging system using 475-nm excitation, an FITC (fluorescein isothiocyanate) filter, and a 200-ms acquisition time for each image. The stack range was $4.5 \mu \mathrm{m}$ with $0.25 \mu \mathrm{m} z$ axis increments (total of 19 images/stack). The statistical significance of distributions was compared with random distributions by $\chi^{2}$ analysis, and Student $t$-tests determined the similarity of zone 1 values.

Time-lapse imaging was performed on a Zeiss LSM510 confocal microscope with three-dimensional (3D) imaging over time, taking six optical slices of 450 nm with a Hyperfine HRZ 200 motor, at 1.5-second time intervals. For high-throughput analysis the 3D stacks were projected onto a single $x, y$ plane by maximum intensity projection prior to analysis, so that data sets from either the Nup49-GFP or the tetR-GFP background essentially represent $2 \mathrm{D}$ projections of $3 \mathrm{D}$ information. $3 \mathrm{D}$ tracking of focus movement in $x, y, z$ after deconvolution was performed with Imaris Time module (Rel 4.0; Bitplane, Zürich). The quantity of data analyzed and the efficiency of our computing algorithms limits our 3D positional analysis for large numbers of time-lapse series.

\section{ACKNOWLEDGMENTS}

We thank T. Laroche, K. Dubrana, G. Van Houwe, and K. Bystricky for thoughtful discussions, technical advice, and experimental support. This work was funded by the NIH (GM51402) and the Swiss National Science Foundation and its NCCR Frontiers in Genetics program. The Novartis Foundation supported M.R.G. while on sabbatical in the Gasser lab.

\section{REFERENCES}

Andrulis E., Neiman A.M., Zappulla D.C., and Sternglanz R. 1998. Perinuclear localization of chromatin facilitates transcriptional silencing. Nature 394: 592.

Andrulis E.D., Zappulla D.C., Ansari A., Perrod S., Laiosia C.V., Gartenberg M R., and Sternglanz R. 2002. Esc1, a nuclear periphery protein required for Sir4-based plasmid anchoring and partitioning. Mol. Cell. Biol. 22: 8292.

Ansari A. and Gartenberg M.R. 1997. The yeast silent information regulator Sir4p anchors and partitions plasmids. Mol.
Cell. Biol. 17: 7061.

Bi X. and Broach J.R. 2001. Chromosomal boundaries in $S$. cerevisiae. Curr. Opin. Genet. Dev. 11: 199.

Boulton S.J. and Jackson S.P. 1998. Components of the Ku-dependent non-homologous end-joining pathway are involved in telomeric length maintenance and silencing. EMBO J. 17: 1819.

Buck S.W. and Shore D. 1995. Action of a RAP1 carboxy-terminal silencing domain reveals an underlying competition between HMR and telomeres in yeast. Genes Dev. 9: 370.

Cheng T.-H., Li Y.-C., and Gartenberg, M.R. 1998. Persistence of an alternate chromatin structure at silenced loci in the absence of silencers. Proc. Natl. Acad. Sci. 95: 5521.

Chung H.M., Shea C., Fields S., Taub R.N., Van der Ploeg L.H., and Tse D.B. 1990. Architectural organization in the interphase nucleus of the protozoan Trypanosoma brucei: Location of telomeres and mini-chromosomes. EMBO J. 9: 2611.

Cockell M. and Gasser S.M. 1999. Nuclear compartments and gene regulation. Curr. Opin. Genet. Dev. 9: 199.

Cormack B.P., Ghori N., and Falkow S. 1999. An adhesin of the yeast pathogen Candida glabrata mediating adherence to human epithelial cells. Science 285: 578.

De Las Penas A., Pan S.J., Castano I., Alder J., Cregg R., and Cormack B.P. 2003. Virulence-related surface glycoproteins in the yeast pathogen C. glabrata are encoded in subtelomeric clusters and subject to RAP1- and SIR-dependent transcriptional silencing. Genes Dev. 17: 2245.

Feuerbach F., Galy V., Trelles-Sticken E., Fromont-Racine M., Jacquier A., Gilson E., Olivo-Marin J.C., Scherthan H., and Nehrbass U. 2002. Nuclear architecture and spatial positioning help establish transcriptional states of telomeres in yeast. Nat. Cell Biol. 4: 214.

Figueiredo L.M., Freitas-Junior L.H., Bottius E., Olivo-Marin J.C., and Scherf A. 2002. A central role for Plasmodium falciparum subtelomeric regions in spatial positioning and telomere length regulation. EMBO J. 21: 815.

Freitas-Junior L.H., Bottius E., Pirrit L.A., Deitsch K.W., Scheidig C., and Scherf A. 2000. Frequent ectopic recombination of virulence factor genes in telomeric chromosome clusters of P. falciparum. Nature 407: 1018 .

Galy V., Olivo-Marin J.-C., Scherthan H., Doye V., Rascalou N., and Nehrbass U. 2000. Nuclear pore complexes in the organization of silent telomeric chromatin. Nature 403: 108.

Gartenberg M.R., Neumann F.R., Laroche T., Blaszczyk M., and Gasser S.M. 2004. Sir-mediated repression can occur independently of chromosomal and subnuclear contexts. Cell 119: 955.

Gasser S.M. and Cockell M.M. 2001. The molecular biology of SIR proteins. Gene 279: 1

Gilson E., Roberge M., Giraldo R., Rhodes D., and Gasser S.M. 1993. Distortion of the DNA double helix by RAP1 at silencers and multiple telomeric binding sites. J. Mol. Biol. 231: 293.

Gotta M., Laroche T., Formenton A., Maillet L., Scherthan H., and Gasser S.M. 1996. The clustering of telomeres and colocalization with Rap1, Sir3, and Sir4 proteins in wild-type $S$. cerevisiae. J. Cell Biol. 134: 1349.

Gottschling D.E., Aparicio O.M., Billington B.L., and Zakian V.A. 1990. Position effect at $S$. cerevisiae telomeres: Reversible repression of Pol II transcription. Cell 63: 751.

Halme A., Bumgarner S., Styles C., and Fink G.R. 2004. Genetic and epigenetic regulation of the FLO gene family generates cell-surface variation in yeast. Cell 116: 405 .

Hecht A., Strahl-Bolsinger S., and Grunstein M. 1996. Spreading of transcriptional repressor SIR3 from telomeric heterochromatin. Nature 383: 92.

Hecht A., Laroche T., Strahl-Bolsinger S., Gasser S.M., and Grunstein M. 1995. Histone H3 and H4N-termini interact with SIR3 and SIR4 proteins: A molecular model for the formation of heterochromatin in yeast. Cell 80: 583 .

Hediger F., Dubrana K., and Gasser S.M. 2002a. Myosin-like proteins 1 and 2 are not required for silencing or telomere anchoring but act in the Tel1 pathway of telomere length control. J. Struct. Biol. 140: 79. 
Hediger F., Taddei A., Neumann F.R., and Gasser S.M. 2004 Methods for visualizing chromatin dynamics in living yeast. Methods Enzymol. 375: 345.

Hediger F., Neumann F.R., Van Houwe G., Dubrana K., and Gasser S.M. 2002b. Live imaging of telomeres: yKu and Sir proteins define redundant telomere-anchoring pathways in yeast. Curr. Biol. 12: 2076.

Heun P., Laroche T., Raghuraman M.K., and Gasser S.M. 2001a. The positioning and dynamics of origins of replication in the budding yeast nucleus. J. Cell Biol. 152: 385.

Heun P., Laroche T., Shimada K., Furrer P., and Gasser S.M. 2001b. Chromosome dynamics in the yeast interphase nucleus. Science 294: 2181

Holmes S.G., Rose A.B., Steuerle K., Saez E., Sayegh S., Lee Y.M., and Broach J.R. 1997. Hyperactivation of the silencing proteins, Sir2p and Sir3p, causes chromosome loss. Genetics 145: 605 .

Hoppe G.J., Tanny J.C., Rudner A.D., Gerber S.A., Danaie S., Gygi S.P., and Moazed D. 2002. Steps in assembly of silent chromatin in yeast: Sir3-independent binding of a Sir2/Sir4 complex to silencers and role for Sir2-dependent deacetylation. Mol. Cell. Biol. 22: 4167.

Jencks W.P. 1975. Binding energy, specificity, and enzymic catalysis: The circe effect. Adv. Enzymol. Relat. Areas Mol. Biol. 43: 219.

Kimura A., Umehara T., and Horikoshi M. 2002. Chromosomal gradient of histone acetylation established by Sas $2 p$ and Sir $2 p$ functions as a shield against gene silencing. Nat. Genet. 32: 370 .

Laroche T., Martin S.G., Tsai-Pflugfelder M., and Gasser S.M 2000. The dynamics of yeast telomeres and silencing proteins through the cell cycle. J. Struct. Biol. 129: 159.

Laroche T., Martin S.G., Gotta M., Gorham H.C., Pryde F.E., Louis E.J., and Gasser S.M. 1998. Mutation of yeast Ku genes disrupts the subnuclear organization of telomeres. Curr. Biol. 8: 653 .

Luo K., Vega-Palas M.A., and Grunstein M. 2002. Rap1-Sir4 binding independent of other Sir, yKu, or histone interactions initiates the assembly of telomeric heterochromatin in yeast. Genes Dev. 16: 1528 .

Lustig A.J., Liu C., Zhang C., and Hanish J.P. 1996. Tethered Sir3p nucleates silencing at telomeres and internal loci in Saccharomyces cerevisiae. Mol. Cell. Biol. 16: 2483.

Maillet L., Boscheron C., Gotta M., Marcand S., Gilson E., and Gasser S.M. 1996. Evidence of silencing compartments within the yeast nucleus: A role for telomere proximity and Sir protein concentration in silencer-mediated repression. Genes Dev. 10: 1796.

Maillet L., Gaden F., Brevet V., Fourel G., Martin S.G., Dubrana
K., Gasser S.M., and Gilson E. 2001. Ku-deficient yeast strains exhibit alternative states of silencing competence. EMBO Rep. 2: 203.

Marcand S., Buck S.W., Moretti P., Gilson E., and Shore D. 1996. Silencing of genes at nontelomeric sites in yeast is controlled by sequestration of silencing factors at telomeres by Rap1 protein. Genes Dev. 10: 1297.

Meneghini M.D., Wu M., and Madhani H.D. 2003. Conserved histone variant $\mathrm{H} 2 \mathrm{~A} . \mathrm{Z}$ protects euchromatin from the ectopic spread of silent heterochromatin. Cell 112: 725.

Mishra K. and Shore D. 1999. Yeast Ku protein plays a direct role in telomeric silencing and counteracts inhibition by Rif proteins. Curr. Biol. 9: 1123.

Moretti P., Freeman K., Coodly L., and Shore D. 1994. Evidence that a complex of SIR proteins interacts with the silencer and telomere-binding protein RAP1. Genes Dev. 8: 2257.

Renauld H., Aparicio O.M., Zierath P.D., Billington B.L., Chhablani S.K., and Gottschling D.E. 1993. Silent domains are assembled continuously from the telomere and are defined by promoter distance and strength, and by SIR3 dosage. Genes Dev. 7: 1133.

Robyr D., Suka Y., Xenarios I., Kurdistani S.K., Wang A., Suka N., and Grunstein M. 2002. Microarray deacetylation maps determine genome-wide functions for yeast histone deacetylases. Cell 109: 437.

Roy R., Meier B., McAinsh A.D., Feldmann H.M., and Jackson S.P. 2004. Separation-of-function mutants of yeast Ku80 reveal a Yku80p-Sir4p interaction involved in telomeric silencing. J. Biol. Chem. 279: 86 .

Scherf A., Figueiredo L.M., and Freitas-Junior L.H. 2001. Plasmodium telomeres: A pathogen's perspective. Curr. Opin. Microbiol. 4: 409.

Strahl-Bolsinger S., Hecht A., Luo K., and Grunstein M. 1997. SIR2 and SIR4 interactions differ in core and extended telomeric heterochromatin in yeast. Genes Dev. 11: 83.

Suka N., Luo K., and Grunstein M. 2002. Sir2p and Sas2p opposingly regulate acetylation of yeast histone $\mathrm{H} 4$ lysine 16 and spreading of heterochromatin. Nat. Genet. 32: 378.

Taddei A., Hediger F., Neumann F.R., Bauer C., and Gasser S.M. 2004. Separation of silencing from perinuclear anchoring functions in yeast $\mathrm{Ku} 80, \mathrm{Sir} 4$ and Esc 1 proteins. EMBOJ. 23: 1301 .

Tham W.H., Wyithe J.S., Ferrigno P.K., Silver P.A., and Zakian V.A. 2001. Localization of yeast telomeres to the nuclear periphery is separable from transcriptional repression and telomere stability functions. Mol. Cell 8: 189.

van Leeuwen F., Gafken P.R., and Gottschling D.E. 2002. Dot1p modulates silencing in yeast by methylation of the nucleosome core. Cell 109: 745. 


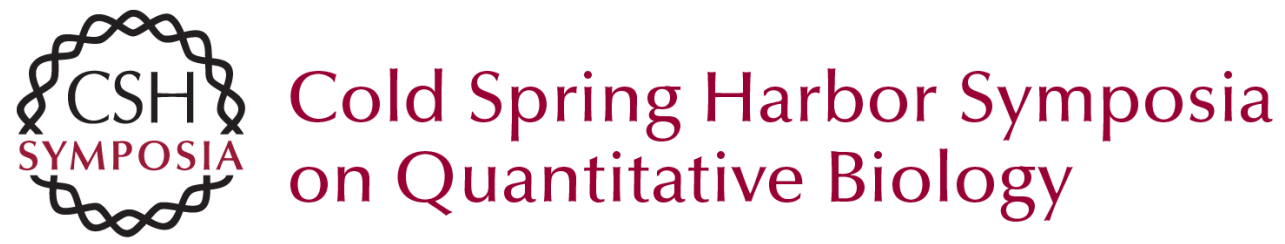

\section{The Function of Telomere Clustering in Yeast: The Circe Effect}

S.M. GASSER, F. HEDIGER, A. TADDEI, et al.

Cold Spring Harb Symp Quant Biol 2004 69: 327-338

Access the most recent version at doi:10.1101/sqb.2004.69.327

References This article cites 51 articles, 22 of which can be accessed free at: http://symposium.cshlp.org/content/69/327.full.html\#ref-list-1

License

Email Alerting Receive free email alerts when new articles cite this article - sign up in Service the box at the top right corner of the article or click here. 